\title{
Gesundheitsversorgung als öffentliche Aufgabe
}

\author{
Das finnische Gesundheitssystem gilt entweder als Vorbild oder als abschreckendes \\ Beispiel - je nach Sichtweise. Es gibt wenig Wahlfreiheit, dafür tiefe Kosten und gute \\ Gesundheitsresultate. Die Stadt Helsinki lud im Februar Vertreterinnen deutschspra- \\ chiger Ärztezeitungen zu einer Besichtigung ihrer staatlichen Gesundheitseinrichtun- \\ gen ein. Die Eindrücke sind zwiespältig, aber mehrheitlich positiv.
}

Anna Sax

lic. oec. publ., MHA, Gesundheitsökonomin und Mitglied der Redaktion
Zwei Dinge gibt es, worauf Finninnen und Finnen auch im Alter niemals verzichten wollen: Sauna und Würste braten im Freien. Deshalb gibt es gleich 16 Saunas für die 200 Bewohner und Nutzerinnen des Kontula-Seniorenzentrums von Helsinki. Und um das Grillfeuer im Garten sitzt gemütlich eine Gruppe von Seniorinnen - warm eingepackt, denn die Temperatur misst 12 Grad minus.

\section{Zu Hause wohnen, so lange wie möglich}

Leila Koivisto, die Leiterin des Seniorenzentrums, führt uns durch das Haus und durch wohnliche Räume. In den 14 Wohngruppen hat jeder sein eigenes Zimmer, dazu gibt es einen Gemeinschaftsraum und eine Küche. Bis es so weit ist und jemand definitiv hier einzieht, dauert es eine Weile. Eigentlich, so Koivisto, will man die Gäste nämlich wieder loswerden. So durchläuft jede neue Bewohnerin zunächst eine mehrwöchige Evaluationsperiode: In dieser Zeit werden die funktionalen Kapazitäten und Rehabilitationsmöglichkeiten abgeklärt, und ein individueller Betreuungsplan wird erstellt. Es geht also erst einmal in die Werkstatt, ins Trainingszentrum und ins Schwimmbad. Sozialberatung und Coaching werden angeboten, auch für die Angehörigen. Ziel ist es immer, die physischen und mentalen Fähigkeiten so weit zu verbessern und zu erhalten, dass die Leute weiter zu Hause leben können. «Hei-hei, tschüs» ruft uns die alte Dame fröhlich hinterher, die gerade aus dem Kraftraum kommt. Sie hat gute Chancen, nochmals in ihre Wohnung zurückzukehren.

Selbständiges Wohnen zu Hause wird vom Kontula-Seniorenzentrum wie auch von allen anderen Zentren gefördert und unterstützt. Soziale Ausgrenzung soll vermieden, funktionale Kapazitäten und Unabhängigkeit sollen erhalten bleiben. Deshalb gibt es eine Tagesstruktur und die Möglichkeit befristeter Aufenthalte, um den Angehörigen Berufstätigkeit und Ferien zu ermöglichen. Es gibt Handarbeitskurse, Gymnastik und Spielnachmittage. Die ambulanten Angebote sind gratis. Wer im Zentrum wohnt, bezahlt $85 \%$ seines Einkommens, das normalerweise aus der Altersrente besteht, für Kost und Logis, Pflege und Betreuung. 200 Angestellte kümmern sich um Bewohner und Gäste. Sie koordinieren auch die Betreuung mit Angehörigen, Spitex und Sozialdiensten.

Die Besucherin aus der Schweiz ist beeindruckt: So sieht es aus, wenn alle am gleichen Strick ziehen. Die Betreuenden unternehmen alles, damit alte und behinderte Menschen zu Hause leben können. Wenn es

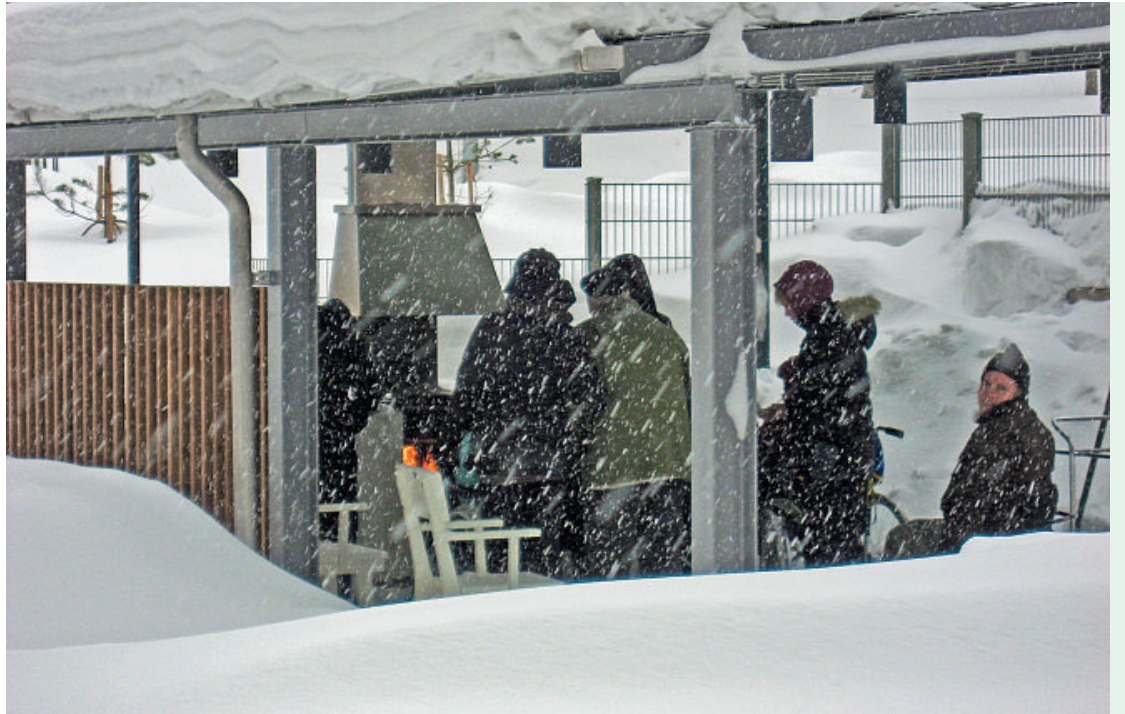

Würste grillen im Freien, auch im Winter bei minus $12 \mathrm{Grad}$ - darauf würden Finnen niemals verzichten. 


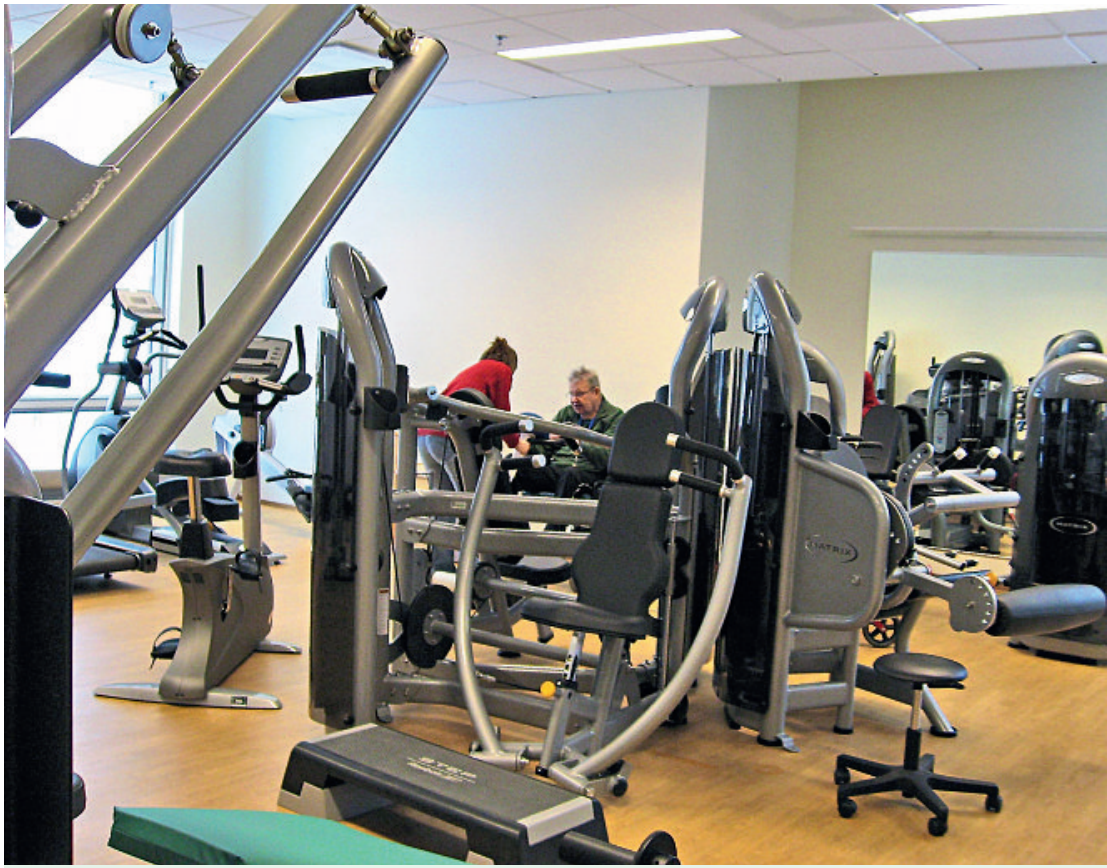

Ein Kraftraum im Altersheim. Ziel ist, dass die Senioren bald wieder nach Hause gehen können. planung, Zahnbehandlung und Spitex-Diensten bis hin zu Sozialarbeit mit Drogenabhängigen. Sie sind erste Anlaufstelle für fast alle Finninnen und Finnen, die medizinische Betreuung suchen. Jeder Bezirk ist zudem Teil einer Spitalregion.

Die Stadt Helsinki, wo ein gutes Zehntel der 5,3 Millionen Finninnen und Finnen lebt, betreibt 26 Gesundheitszentren, 39 Zahnkliniken, fünf Stadtspitäler, ein Psychiatriespital sowie ein breites Angebot an Spitex und ambulanten psychiatrischen Diensten. Zudem steht hier das grösste der fünf Universitätsspitäler des Landes. Im Unterschied zu den meisten ländlichen Gebieten gibt es in Helsinki auch ein privates Angebot mit drei Spitälern und mehreren Polikliniken. Viele Ärztinnen und Ärzte sind im öffentlichen Sektor teilzeitlich beschäftigt und arbeiten daneben noch auf eigene Rechnung im Privatsektor. Wer sich privat behandeln lassen will, bezahlt $60 \%$ der Kosten aus der eigenen Tasche. Der Rest wird über die öffentliche Krankenversicherung gedeckt.

Wir besuchen das Gesundheitszentrum Kallio im Süden der Stadt. Als erstes fällt mir die Kabine auf, wo sich die Patientinnen beim Eintreffen gleich selber den Blutdruck messen. Würde das hierzulande nicht schon fast als Zumutung empfunden? Aber weshalb eigentlich ist es in der Schweiz selbstverständlich, dass die Ärztin oder zumindest eine Praxisassistentin diese einfache Handreichung vornimmt? Tuula Kauppinen, die leitende Oberschwester des Gesundheitszentrums, hat nur ein müdes Lächeln übrig für meine Ahnungslosigkeit im Umgang mit einem Blutdruckmessgerät. Der Arzt kommt sowieso erst dann zum Einsatz, wenn es ihn braucht. Das heisst, wenn die Pflegefachfrau im Vorzimmer entscheidet, dass ein Arztbesuch notwendig sei. Übrigens kann sich, wer in Finnland wohnt und nicht privatversichert ist, weder das Gesundheitszentrum noch die Hausärztin bzw. den Hausarzt selber auswählen. Man wird je nach Wohnort zugeteilt. Was in der Schweiz einen Aufstand auslösen würde, ist hier selbstverständlich und von einem Grossteil der Bevölkerung akzeptiert.

\section{Gute Gesundheitsresultate bei geringen Kosten}

Die finnische Bevölkerung ist insgesamt weder gesünder noch ungesünder als in anderen Ländern in vergleichbaren wirtschaftlichen Verhältnissen. Mit einer durchschnittlichen Lebenserwartung von 79,5 Jahren liegt Finnland im Mittelfeld der OECD-Länder (Die Schweiz liegt mit 81,9 Jahren hinter Japan an zweiter Stelle), wobei der Unterschied zwischen den Geschlechtern auffallend gross ist (Männer 76 Jahre, Frauen 83 Jahre). Das könnte eine Folge sein des unter Männern verbreiteten Alkoholmissbrauchs, wie Riitta Simoila, Entwicklungsleiterin des Gesundheitszentrums, vermutet. Bei der Säuglingssterblichkeit schneidet dafür Finnland, das über ein gut ausgebautes Mutter-Kind-Gesundheitsnetz verfügt, überdurchschnittlich und deutlich besser ab als die Schweiz. Programmen über Gesundheitsförderung, Familienwird aus Lohnprozenten und Steuern alimentiert, Angebotsplanung und Grundversorgung erfolgen durch die 340 Bezirke. Die Gesundheitszentren der Bezirke bieten neben der medizinischen Grundversorgung eine breite Palette von Dienstleistungen an, von Screening-

sein muss, schaut die Spitex 5-6-mal täglich vorbei. Heim. Das Seniorenzentrum will es so, die Stadt Helsinki, der finnische Staat. Die politische Linie ist klar. Macht das die Menschen glücklich? Schwer zu sagen. ihnen $\mathrm{zu}$ reden ist unmöglich.

\section{Konsens für Staatsmedizin}

genden Sprache, ist hierzulande vor allem bekannt als Wer fur Langläufer und als Schauplatz von Kin hier Argumente für und gegen eine staatlich gesteuerte Gesundheitsversorgung. Sicher ist, dass das finnische Gesundheitswesen das Produkt ist eines starken Sozialwird. «Staatsmedizin», bei uns quasi zum Schimpfwort sorgt für seine Bevölkerung, und diese bezahlt im Gegibt es wenig Raum für Eigenverantwortung. Kein Vetsicherungswechsel, keine Arztwahl, beschränkte pri. Angebote. Selbst dem Staat grundsätzlich woh vermutlich ihre Mühe mit der weitgehenden mit der weitgehenden Abse on Wahlfreiheit.

Die staatliche Krankenversicherung in Finnland 
Die starke staatliche Steuerung und Einschränkung der Wahlfreiheit schlägt sich in moderaten Kosten nieder. Nur gerade 2840 US-Dollar pro Kopf kostete 2007 die Gesundheitsversorgung in Finnland, gegenüber 4417 US-Dollar in der Schweiz. Das ist ein Mehraufwand von $55 \%$, den wir in der Schweiz betreiben, und für den wir zwar nicht wesentlich mehr Gesundheit, dafür mehr Wahlfreiheit, kürzere Wartezeiten, aufwendigere Behandlungen und bessere Löhne für das Gesundheitspersonal erkaufen.

\section{Wartezeiten und Personalknappheit}

Die Wartezeiten sind in Finnland ein Thema, wie uns Riitta Lehtonen, die Kommunikationschefin des Universitätsspitals, bestätigt. Zwar verabschiedete die Regierung vorletztes Jahr ein Gesetz, das die Wartezeit für Wahloperationen auf höchstens sechs Monate beschränkt. Spitäler können mit hohen Bussen bestraft werden, wenn sie diese Frist überschreiten. Bevor das neue Gesetz in Kraft trat, befanden sich in Helsinki 20000 Personen auf der Warteliste, davon 1800 seit mehr als sechs Monaten. Unter der Androhung von Bussen gelang es tatsächlich, die Warteliste deutlich abzubauen. Dies hatte allerdings den Effekt, dass die Zahl der Leute zurückging, die eine Privatversicherung abschlossen, um die Wartezeiten zu verkürzen. Damit nahmen wieder mehr Patienten die Leistungen der öffentlichen Hand in Anspruch. Die Folge davon ist ein erneutes Anwachsen der Warteliste.

Auch Maila Malinen, Patientin im Gesundheitszentrum und Mutter von drei Kindern, ärgert sich manchmal über lange Wartezeiten. Und doch würde es ihr nicht einfallen, sich privatzuversichern. «Im Notfall sind wir immer rasch und in guter Qualität versorgt worden», beschreibt sie ihre Erfahrungen. Sie habe auch schon einen Neurologen privat aufgesucht und einen Teil der Kosten aus der eigenen Tasche bezahlt. Eine Privatversicherung jedoch, findet Frau Malinen, lohne sich nicht. «Wenn es wirklich darauf ankommt, funktioniert das staatliche System.»

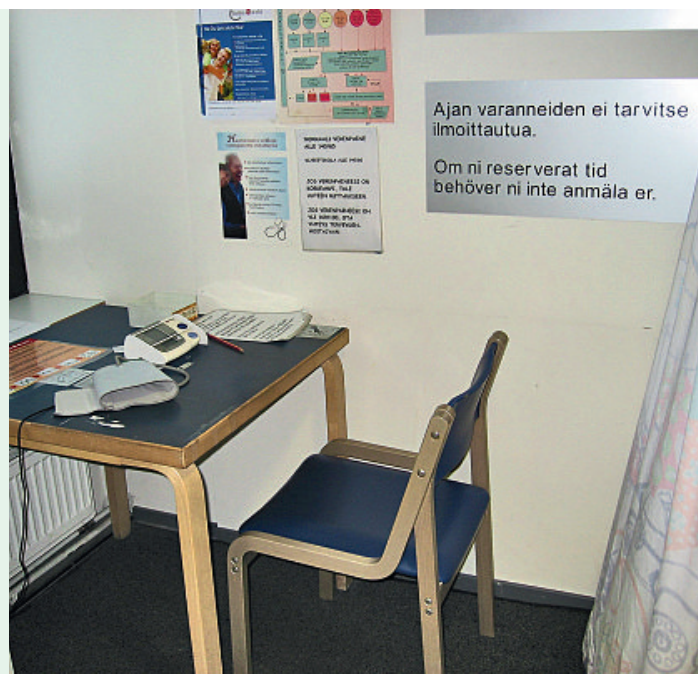

Ein vieldiskutiertes Thema sind die Arbeitsbedingungen und Kompetenzen des Gesundheitspersonals. Pflegefachfrauen übernehmen in Finnland viele Aufgaben der Grundversorgung, seit kurzem können sie auch Medikamente verschreiben. Personalmangel ist auch hier ein Thema. Inzwischen sind 15\% der Pflegefachkräfte ausländischer Herkunft, viele kommen aus den Nachbarländern Russland und Estland. Mit ca. 2600 Euro pro Monat entspricht der Lohn der Pflegefachpersonen in etwa dem Landesdurchschnitt. Mit einer 37-Stunden-Woche, langen Ferien und Programmen gegen Stress und Burnout wird versucht, das Personal möglichst lange bei der Stange zu halten.

\section{«Wenn es wirklich darauf an- kommt, funktioniert das staatliche System»}

Weniger virulent als in der Schweiz scheint der Mangel an Allgemeinärzten und -ärztinnen zu sein. Insgesamt gebe es genügend Ärzte, versichert die Verantwortliche für medizinische Ausbildung an der medizinischen Fakultät der Universität Helsinki, Anne Pitkäranta. 600 Studienplätze werden pro Jahr vergeben, verteilt auf die fünf Universitätsspitäler - gleich viele wie in der Schweiz bei einer deutlich kleineren Bevölkerungszahl. Das Problem liegt, wie anderswo auch, in der geographischen Verteilung. Mangel gibt es - was wenig erstaunlich ist - auf dem Land. Gesundheitszentren in ländlichen Gebieten, die keine Ärztin finden, gelangen an private Vermittlungsfirmen, die Ärzte «vermieten» zu Tarifen, die deutlich höher sind als die Löhne der angestellten Ärzte. So kommt es vor, dass eine junge, privat vermittelte Ärztin mehr verdient als die erfahrene, aber staatlich entlohnte Leiterin eines Gesundheitszentrums.

\section{Kein Paradies, aber praxistauglich}

Wer sich im Gesundheitswesen Wettbewerb und Wahlfreiheit wünscht, ist in Finnland am falschen Ort. Wer klare politische Ziele, Steuerung und flächendeckende Versorgung sowohl stationär wie auch ambulant befürwortet, kommt schon eher auf seine Rechnung. Der finnische Staat sorgt zusammen mit den Bezirken dafür, dass die Gesundheitsversorgung für alle gewährleistet ist. Dazu gehören auch Aufgaben wie die Erhaltung und Förderung der Selbständigkeit und die Entlastung der Angehörigen. Einen hohen Stellenwert hat zudem Prävention und Gesundheitsförderung. Es scheint, dass der finnische Hang zum Pragmatismus sich auch im Gesundheitswesen niederschlägt: Alle bekommen, was sie brauchen, um den Alltag möglichst reibungslos bewältigen zu können. Das Modell

ist eindeutig praxistauglich.
In dieser Kabine messen ins Gesundheitszentrum von Helsinki selbst ihren Blutdruck. 